PROCEEDINGS OF THE

AMERICAN MATHEMATICAL SOCIETY

Volume 127, Number 12, Pages 3527-3532

S 0002-9939(99)05531-8

Article electronically published on August 5, 1999

\title{
UNIQUENESS THEOREM FOR A CAUCHY PROBLEM WITH HYSTERESIS
}

\author{
JANA KOPFOVÁ
}

(Communicated by Hal L. Smith)

\begin{abstract}
The Cauchy problem for an ordinary differential equation coupled with a hysteresis operator is studied. Under physically reasonable assumptions on the forcing term, uniqueness of solutions is shown without assuming Lipschitz continuity of the hysteresis curves. The result is true for any kind of hysteresis operators with monotone curves of motion.
\end{abstract}

\section{INTRODUCTION}

In this paper we consider the question of uniqueness of solutions for the Cauchy problem for an ordinary differential equation coupled with a hysteresis operator without assuming its Lipschitz continuity. By "solution" we mean a continuous, differentiable function, which satisfies (2) and satisfies (1) on some right neighborhood of $t=0$. The equation we consider is

$$
\begin{aligned}
\frac{d u}{d t}+\mathcal{F}(u) & =f \quad \text { in }(0, T), \\
u(0) & =u_{0} .
\end{aligned}
$$

Here $\mathcal{F}$ represents a hysteresis operator: $C([0, T]) \rightarrow C([0, T])$ and we assume $f$ continuous on $[0, T]$. The existence of a solution of (1)-(2) is well known; see [5]. As pointed out by Visintin (see [4], p.324), uniqueness was an open problem for $\mathcal{F}$ not Lipschitz continuous, while it was known that Lipschitz continuity guarantees uniqueness. Using simple techniques from the theory of differential equations, we show that under physically reasonable assumptions on $f$ we do have uniqueness even in the non-Lipschitz case. This is done first for $f=0$, then extended to the more general case. As was shown recently by V.Chernorutskii and D.Rachinskii in [1], additional assumptions are needed in the non-Lipschitz case. In [1] they constructed a specific continuous right hand side, oscillatory in every neighborhood of 0 , for which there is nonuniqueness.

\section{Preliminaries}

In this section we state a uniqueness theorem for the Cauchy problem without the hysteresis operator, which will be useful in the sequel. The result is classical, a detailed proof is given in [2], Section III.6.

Received by the editors October 11, 1996.

1991 Mathematics Subject Classification. Primary 34A12; Secondary 34A60.

(C)1999 American Mathematical Society 
Theorem 1. Let $U(t, u)$ be a continuous real-valued function for $t_{0} \leq t \leq t_{0}+a$, $\left|u-u_{0}\right| \leq b$, which is nonincreasing with respect to $u$ (for fixed $t$ ). Then the initial value problem

$$
\begin{aligned}
\frac{d u}{d t} & =U(t, u), \\
u\left(t_{0}\right) & =u_{0}
\end{aligned}
$$

has at most one solution on any interval $\left[t_{0}, t_{0}+\epsilon\right], \epsilon>0$.

\section{Hysteresis OPERATORS}

The term hysteresis means to lag behind. Hysteresis operators were first systematically studied by M.A.Krasnosel'skii and A.V.Pokrovskii; see their monograph, [3].

When speaking of hysteresis, one usually refers to a relation between two scalar time-dependent quantities $u(t)$ and $w(t)$ that cannot be expressed in terms of a single valued function, but takes the form of loops, like the one depicted in Figure 1. When the pair $(u, w)$ is on the curve $\gamma_{r}$ and $u$ increases, then the pair $(u, w)$ moves along the curve $\gamma_{r}$. If the pair $(u, w)$ is on the curve $\gamma_{l}$ and $u$ decreases, then the pair $(u, w)$ moves on the curve $\gamma_{l}$. Moreover, when $u$ inverts its movement, the pair $(u, w)$ moves into the interior of the region bounded by the curves $\gamma_{l}$ and $\gamma_{r}$, this behavior must be described by the specific model.

In this section we will restrict ourselves to the definition of the generalized play operator, as used by Visintin. For more details as well as for definitions of other hysteresis operators, see [4] or [3].

Let

$$
\gamma_{l}, \gamma_{r}: \mathbb{R} \rightarrow \mathbb{R} \text { be continuous nondecreasing functions with } \gamma_{r} \leq \gamma_{l} \text {. }
$$

Now, given $w_{0} \in \mathbb{R}$, we construct the hysteresis operator $\mathcal{E}\left(\cdot, w_{0}\right)$ as follows. Let $u$ be any continuous, piecewise linear function on $[0, T]$ such that $u$ is linear on

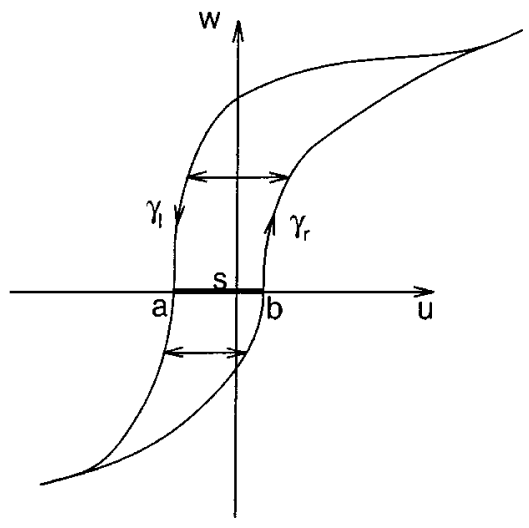

Figure 1. A generalized play with boundary curves $\gamma_{l}$ and $\gamma_{r}$. 
$\left[t_{i-1}, t_{i}\right]$ for $i=1,2, \ldots, N$. We then define $w:=\mathcal{E}\left(u, w_{0}\right):[0, T] \rightarrow \mathbb{R}$ by

$$
w(t):= \begin{cases}\min \left\{\gamma_{l}(u(0)), \max \left\{\gamma_{r}(u(0)), w_{0}\right\}\right\} & \text { if } t=0, \\ \min \left\{\gamma_{l}(u(t)), \max \left\{\gamma_{r}(u(t)), w\left(t_{i-1}\right)\right\}\right\} & \text { if } t \in\left(t_{i-1}, t_{i}\right], i=1,2, \ldots, N .\end{cases}
$$

Note that $w(0)=w_{0}$ only if $\gamma_{r}(u(0)) \leq w_{0} \leq \gamma_{l}(u(0))$.

As proved in Visintin [4], Sect.III.2, for any continuous piecewise linear functions $u_{1}, u_{2}$ on $[0, T]$, with the notation $\epsilon_{k}:=\mathcal{E}\left(u_{k}, w_{0 k}\right), k=1,2$, we have the following inequality:

$$
\begin{aligned}
\max _{\left[t_{1}, t_{2}\right]}\left|\epsilon_{1}-\epsilon_{2}\right| & \leq \max \left\{\left|\epsilon_{1}\left(t_{1}\right)-\epsilon_{2}\left(t_{1}\right)\right|, m_{M}\left(\max _{\left[t_{1}, t_{2}\right]}\left|u_{1}-u_{2}\right|\right)\right\} \\
\forall\left[t_{1}, t_{2}\right] & \subset[0, T],
\end{aligned}
$$

where for any continuous function $f: \mathbb{R} \rightarrow \mathbb{R}$ and any constant $M>0,|f|_{M}(h)$ denotes its local modulus of continuity:

$$
\begin{gathered}
|f|_{M}(h):=\sup \left\{\left|f\left(y_{1}\right)-f\left(y_{2}\right)\right|: y_{1}, y_{2} \in[-M, M],\left|y_{1}-y_{2}\right| \leq h\right\} \quad \forall h>0, \\
m_{M}(h):=\max \left\{\left|\gamma_{l}\right|_{M}(h),\left|\gamma_{r}\right|_{M}(h)\right\} \quad \forall h, M>0,
\end{gathered}
$$

and

$$
M:=\max \left\{\left|u_{k}(t)\right|: t \in[0, T], k=1,2\right\} .
$$

Hence $\mathcal{E}\left(\cdot, w_{0}\right)$ has a unique continuous extension, denoted by $\mathcal{E}\left(\cdot, w_{0}\right)$ again, to an operator

$$
\mathcal{E}: C([0, T]) \times \mathbb{R} \rightarrow C([0, T]) .
$$

This operator is called a generalized play; see Figure 1.

The inequality (6) holds also for this extended operator, which is then uniformly continuous on bounded sets. If $\gamma_{l}, \gamma_{r}$ are Lipschitz continuous, then $\mathcal{E}$ is also Lipschitz continuous.

The generalized play operator also satisfies the piecewise monotone property, a property very often satisfied by hysteresis operators, i.e.

$$
\left\{\begin{array}{l}
\forall\left(u, w_{0}\right) \in \operatorname{Dom}(\mathcal{F}), \forall\left[t_{1}, t_{2}\right] \subset[0, T] \\
\text { if } u \text { is nondecreasing (resp. nonincreasing) in }\left[t_{1}, t_{2}\right] \\
\text { then so is } \mathcal{F}\left(u, w_{0}\right) .
\end{array}\right.
$$

\section{Main Result}

Using the theorem from Section 2, we prove a uniqueness theorem for (1)-(2), when $\mathcal{F}$ is a generalized play operator.

Theorem 2. Suppose that $\mathcal{F}\left(u, w_{0}\right)$ is a generalized play operator with hysteresis boundary curves $\gamma_{l}$ and $\gamma_{r}$, which are strictly increasing, and $f(t) \equiv 0$. Then the solution of the Cauchy problem (1)-(2) is unique.

Proof. From (1) we have

$$
\frac{d u}{d t}=-\mathcal{F}(u)
$$

thus $\frac{d u}{d t}$ is continuous if $u$ solves (1). 
Suppose that $w(0)=0$. Then

$$
\frac{d u}{d t}(0)=0
$$

and therefore all the points

$$
\mathcal{S}=\left\{(u, w) ; w=0, a \leq u \leq b, \text { where } \gamma_{l}(a)=0 \text { and } \gamma_{r}(b)=0\right\}
$$

are equilibria. The solution of (1)-(2) with $w(0)=0$ is unique, $u(t) \equiv u_{0}$. This can be proved as follows: Assume that for some $t_{1}>0$ we have $w\left(t_{1}\right)>0$ and put $t_{0}:=\max \left\{t \in\left[0, t_{1}\right], w(t)=0\right\}$. Then $w(t)>0$ on $\left(t_{0}, t_{1}\right]$, hence in $\left[t_{0}, t_{1}\right]$ the function $u$ is decreasing, therefore $w$ is nonincreasing, which is a contradiction. Similarly for $w\left(t_{1}\right)<0$.

Suppose now $w(0)>0$. Then at $\left(u_{0}, w(0)\right)$

$$
\frac{d u}{d t}(0)=-w(0)<0
$$

thus $u$ is decreasing on a right neighborhood of $t=0$. We have three possibilities. Either $\left(u_{0}, w(0)\right)$ lies inside the hysteresis region or on $\gamma_{r}$ or on $\gamma_{l}$. In the first two cases $\mathcal{F}$ stays constant on some interval $\left[0, t_{1}\right)$; from (12) we have $u=-w(0) t+c_{0}$ and since $u(0)=u_{0}, u=-w(0) t+u_{0}$ for $t \in\left[0, t_{1}\right]$, and $u$ is decreasing until $(u, w)$ hits the hysteresis boundary $\gamma_{l}$. The second possibility is that $\left(u_{0}, w(0)\right)$ lies on $\gamma_{l}$. Here we again have from (12) that $u$ is decreasing and $(u, w)$ moves on the curve $\gamma_{l}$. Therefore $u$ must satisfy the equation

$$
\begin{aligned}
\frac{d u}{d t}+\gamma_{l}(u) & =0, \\
u(0) & =u_{0}
\end{aligned}
$$

which by Theorem 1 has a unique solution and is approaching the equilibrium $(a, 0)$ as $t \rightarrow \infty$. The case $w(0)<0$ can be handled analogously, using the uniqueness of solutions to the problem:

$$
\begin{aligned}
\frac{d u}{d t}+\gamma_{r}(u) & =0, \\
u(0) & =u_{0} .
\end{aligned}
$$

Remark 1. The assumption about strict monotonicity of $\gamma_{l}, \gamma_{r}$ in the statement of Theorem 2 was used only for simplicity and can be easily omitted.

Remark 2. From the above analysis we are actually able to prove more: Except in the trivial case when $w(0)=0$, the set of two solutions $\left\{u_{1}(t) \equiv a, w_{1} \equiv 0 ; u_{2}(t) \equiv\right.$ $\left.b, w_{2} \equiv 0\right\}$ is an attractor; solutions of (1)-(2) are stable and they converge to this set as $t \rightarrow \infty$, depending on the initial value $w(0)$.

Remark 3. Using the same methods as above we can prove uniqueness for (1)-(2) for $\mathcal{F}$ a generalized Prandtl-Ishlinskii operator of play type or any other hysteresis operator, e.g., Preisach, with monotone curves of motion. In this case all the points $\left(u_{0}, 0\right), a \leq u_{0} \leq b$, are equilibria, where $\gamma_{l}(a)=\gamma_{r}(b)=0$, and $\gamma_{l}, \gamma_{r}$ are the hysteresis boundary curves.

Theorem 3. Suppose there exists an $\epsilon>0$ such that $f(t)>w(0)$ (or $f(t)<w(0)$ ) on $[0, \epsilon)$. Then there exists an $\tilde{\epsilon}>0$, such that there exists at most one solution of $(1)-(2)$ on $[0, \widetilde{\epsilon})$. 


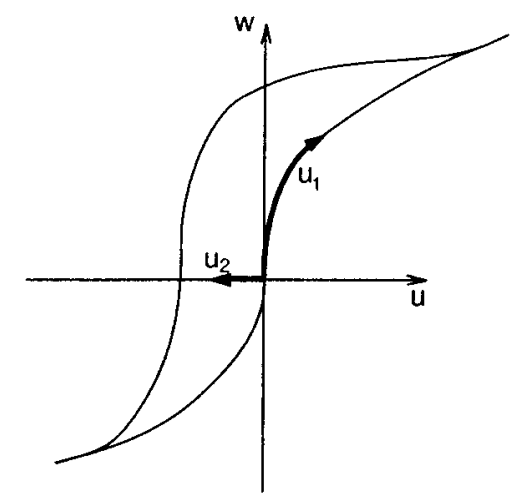

Figure 2. The two solutions in the proof of Theorem 3.

Proof. It follows from the assumptions that there exists an $\widetilde{\epsilon}>0$ such that $f(t)>$ $w(t)$ (or $f(t)<w(t)$ ) on $[0, \widetilde{\epsilon})$. This means that $\frac{d u}{d t}$ does not change sign on $[0, \widetilde{\epsilon})$.

The proof proceeds by contradiction. Let there exist two different solutions on $[0, \widetilde{\epsilon})$. We can assume without loss of generality that $w(0)=0, u_{0}=0$ and that $\gamma_{r}(u)$ is such that $\gamma_{r}(0)=0$ and that the point $(0,0)$ is a point where the curve $\gamma_{r}(u)$ is non-Lipschitz. For $0 \leq t<\widetilde{\epsilon}$, the motions $\left(u_{i}(t), w_{i}(t)\right), i=1,2$, must lie respectively on one of the curves $w=\gamma_{r}(u)$ or $w=0$; see Figure 2. This means that each $u_{i}(t)$ must satisfy either

$$
\frac{d u}{d t}=-\gamma_{r}(u)+f(t)
$$

or

$$
\frac{d u}{d t}=-w_{0}+f(t)=f(t)
$$

for $0 \leq t<\tilde{\epsilon}$. But each of these has a unique solution by Theorem 1 , so we can assume $u_{1}$ solves (13) and $u_{2}$ solves (14).

Look carefully at what this means :

$$
\begin{array}{lll}
\text { for } u_{1}: & \frac{d u_{1}}{d t}=-\gamma_{r}\left(u_{1}\right)+f(t) \geq 0, & \text { i.e. } f(t) \geq \gamma_{r}\left(u_{1}\right)>0, \\
\text { for } u_{2}: & \frac{d u_{2}}{d t}=f(t) \leq 0, & \text { i.e. } f(t) \leq 0,
\end{array}
$$

a contradiction.

Remark 4. As the above proof suggests, we can actually assume less than $f(t)$ has constant sign on $[0, \epsilon)$. It suffices to have assumptions which will guarantee that the solution pair $(u(t), w(t))$ will move either on $\gamma_{r}(u)$ or inside the hysteresis region, on the line $w=0$ (see Figure 3). A little thought shows that we need to exclude the only other alternative to the motions depicted in Figure 2: in every interval $(0, \epsilon)$ the motion leaves $\gamma_{r}(u)$ and returns to it via a horizontal segment; see Figure 3 .

In this case there would exist a sequence $t_{n} \rightarrow 0$, with $Q_{n}=\left(u\left(t_{2 n}\right), w\left(t_{2 n}\right)\right)$ a minimum of $u(t)$ on $\gamma_{r}$ and $P_{n}=\left(u\left(t_{2 n+1}\right), w\left(t_{2 n+1}\right)\right)$ a maximum of $u(t)$ on the 


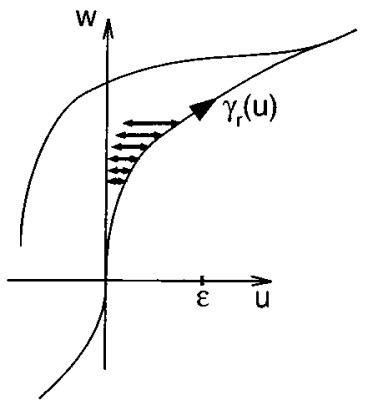

Figure 3. The case when nonuniqueness can occur.

horizontal segment, $t_{2 n+1}<t_{2 n}$. Then we must have

$$
f\left(t_{2 n+1}\right)=\gamma_{r}\left(u\left(t_{2 n}\right)\right)=f\left(t_{2 n}\right) .
$$

This is basically the case from the paper of V. Chernorutskii and D.Rachinskii, [1], when nonuniqueness occurs. This can be excluded, for example, by assuming that $f^{\prime}$ exists on $[0, \epsilon)$, is continuous at $t=0$ and $f^{\prime}(0) \neq 0$.

\section{ACKNOWLEDGEMENT}

This research was supported in part by a grant from the Natural Sciences and Engineering Research Council of Canada.

\section{REFERENCES}

[1] V.Chernorutskii and D.Rachinskii, On uniqueness of an initial-value problem for ODE with hysteresis, Nonl. Diff. Equ. Appl. 4, 1997, 391-399. MR 98j:34004

[2] P.Hartman, Ordinary Differential Equations, Birkhäuser, 1982. MR 83e:34002

[3] M.A.Krasnosel'skii and A.V.Pokrovskii, Systems with Hysteresis, Springer-Verlag, Berlin, 1989. MR 90a:93001

[4] A.Visintin, Differential Models of Hysteresis, Springer-Verlag, Berlin, 1995. MR 96h:47001

[5] A.A.Vladimirov, M.A.Krasnosel'skii, and V.V.Chernorutskii, The Cauchy problem for systems with hysteresis, Russian Acad. Sci. Dokl. Math. 48 (1994), 502-506. MR 94k:47104

Department of Mathematical Sciences, University of Alberta, Edmonton, Alberta, Canada T6G 2G1

E-mail address: kopf@phys.ualberta.ca

Current address: Slezska Univerzita, Matematicky ustav, Bezrucovo nam. 13, 74601 Opava, Czech Republic

E-mail address: jana.kopfova@math.slu.cz 